\title{
Theory of Zero-Resistance States Generated by Radiation in GaAs/AlGaAs
}

\author{
Shigeji Fujita ${ }^{1}$, Kei Ito ${ }^{2}$, Akira Suzuki ${ }^{3 *}$ \\ ${ }^{1}$ Department of Physics, University at Buffalo, Buffalo, USA \\ ${ }^{2}$ Research Division, National Center for University Entrance Examinations, Tokyo, Japan \\ ${ }^{3}$ Department of Physics, Faculty of Science, Tokyo University of Science, Tokyo, Japan \\ Email: ${ }^{*}$ asuzuki@rs.kagu.tus.ac.jp
}

Received May 5, 2012; revised June 10, 2012; accepted July 1, 2012

\begin{abstract}
Mani observed zero-registance states similar to those quantum-Hall-effect states in GaAs/AlGaAs but without the Hall resistance plateaus upon the application of radiations [R. G. Mani, Physica E 22, 1 (2004)]. An interpretation is presented. The applied radiation excites "holes". The condensed composite (c)-bosons formed in the excited channel create a superconducting state with an energy gap. The supercondensate suppresses the non-condensed c-bosons at the higher energy, but it cannot suppress the c-fermions in the base channel, and the small normal current accompanied by the Hall field yeilds a $B$-linear Hall resistivity.
\end{abstract}

Keywords: Superconducting (Zero-Resistance) States; Composite-Boson (Fermion); B-Linear Hall Resistivity; Phonon Exchange

\section{Introduction}

In 2002 Mani et al. [1] observed a set of zero-resistance (superconducting) states in $\mathrm{GaAs} / \mathrm{AlGaAs}$ heterojunction subjected to radiations at the low temperatures $(\sim 1.5 \mathrm{~K})$ and the relatively low magnetic fields $(\sim 0.2 \mathrm{~T})$. Figure 1 represents the data obtained by Mani [2,3] for the Hall $\left(R_{x y}\right)$ and diagonal $\left(R_{x x}\right)$ resistances in GaAs/AlGaAs at $50 \mathrm{GHz}$ and $0.5 \mathrm{~K}$. The resistance $R$ rises exponentially and symmetrically on both sides of the fields centered at

$$
B=\frac{4}{5} B_{f}, \frac{4}{9} B_{f}, \quad B_{f}=\frac{\omega m}{e},
$$

with $\omega=$ radiation frequency, $m=$ effective mass, $e=$ electron charge, indicating the superconducting state with an energy gap $\varepsilon_{g}$ in the elementary excitation spectrum. The phenomenon is similar to that observed in the same system in the traditional quantum Hall effect (QHE) regime ( $T \sim 1.5 \mathrm{~K}, B \sim 10 \mathrm{~T})$ [4] with the main difference that the superconducting states are not accompanied by the Hall resistivity plateaus for the system subjected to radiation, see Figures 1(a) and (b). We call the temperature below which the superconducting state appears the critical temperature $T_{\mathrm{c}}$. The critical temperature $T_{\mathrm{c}}$ $(1.3 \mathrm{~K})$ observed at $B=4 / 5 B_{f}$ is considerably higher than the traditional QHE critical temperature $(0.5 \mathrm{~K})$.

${ }^{*}$ Corresponding author.
Zudov et al. [5] reported similar magnetotransport properties for the system subjected to radiations with slightly different experimental conditions. They suggested that the principal resistivity minima occur at

$$
B=\frac{2}{2 j+1} B_{f}, \quad j=1,2, \cdots,
$$

rather than $B=4 /(4 j+1) B_{f}$ (Mani's case). They also noted a noticeable side resistivity minimum besides the principal set of the minima.

In finer analysis Mani et al. [6] observed, see Figure 2, that (a) the deviation in the Hall resistance

$$
\Delta R_{\mathrm{H}} \equiv R_{\mathrm{H}}-R_{\mathrm{H}, \mathrm{dark}},
$$

correlates with the resistance $R$ such that $\Delta R_{\mathrm{H}}$ nearly vanishes when $R=0$, and (b) $\Delta R_{\mathrm{H}}$ is negative, and it is antisymmetric with respect to small $B$-fields:

$$
\Delta R_{\mathrm{H}}=\alpha B, \quad \alpha=\text { constant, for small } B .
$$

The property (b) means that there is a current due to "hole"-like particles having the charge of the opposite sign to that of the majority ("electron"-like) current carrier. In other words the applied radiation generates the "holes". This may be checked by applying the circularly polarized lasers, which can excite "electrons" or "holes" selectively, depending on the sense of the circular polarization. The small slope $\mathrm{d} \Delta R_{\mathrm{H}} / \mathrm{d} B=\alpha$ means that the "hole" density is considerable higher than the "electron" 


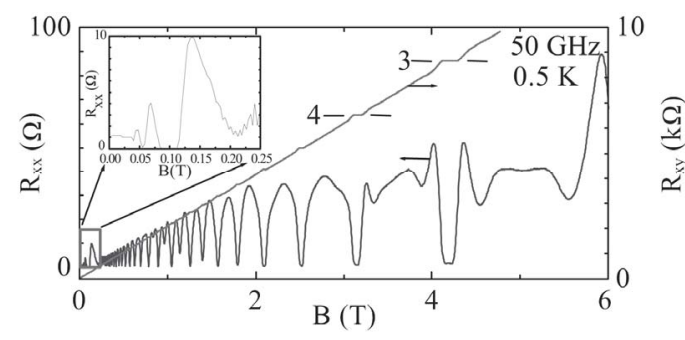

(a)

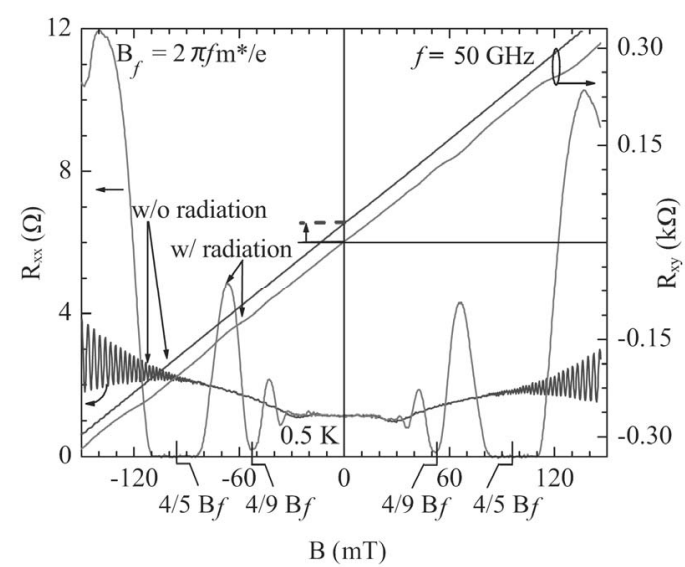

(b)

Figure 1. (a) The Hall $\left(R_{x y}\right)$ and diagonal $\left(R_{x x}\right)$ resistances in a GaAs/AlGaAs under radiation at $50 \mathrm{GHz}$ after Mani [2]. Quantum Hall effects (QHE) occur at high $B$ as $R_{x x}$ vanishes. Inset; An expanded view of the low $B$ data; (b) Data over low magnetic fields obtained both with $(w /)$ and without $(w / o)$ radiation at $50 \mathrm{GHz}$. Here, radiation induced vanishing resistance around $(4 / 5) B_{f}$ does not induce plateaus in the Hall resistance, unlike in QHE. Yet, there are clear microwave induced oscillations in the Hall effect.

density.

Mani et al. [1] suggested for the cause of the spectral gap the electron pairing due to the excitons induced by radiation. Other mechanisms were proposed by several theoretical groups [7-11]. But none of them are conclusive. In particular no explanation is given to the question why the superconducting state can occur without the Hall resistivity plateau.

Earlier Fujita et al. [12] developed a microscopic theory of the QHE based on the electron (fluxon)-phonon interaction. In this theory the composite (c-)particles (bosons or fermions) having a conduction electron and a number of flux quanta (fluxons) are bound by the phonon-exchange attraction. The composite moves as a boson (fermion) according to whether it contains the odd (even) number of fluxons in it. At the Landau Level (LL) occupation number (filling factor) $v=1 / Q, Q$ odd, the c-bosons, each with $Q$ fluxons, are generated, and condense below the critical temperature $T_{c}$. The Hall resistivity plateau is due to the Meissner effect, as explained below.

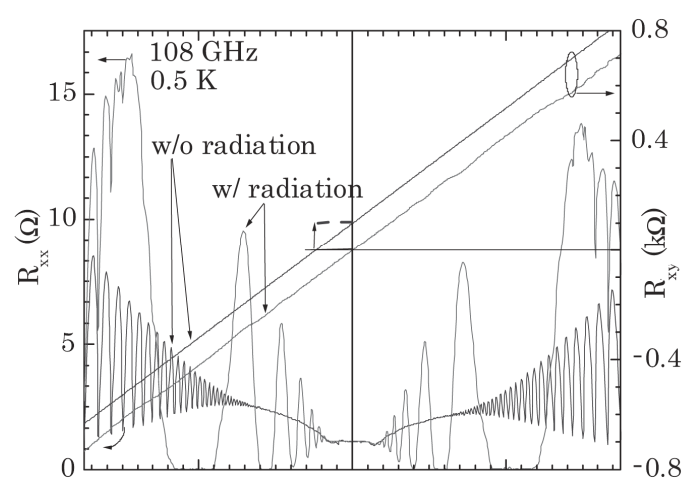

(a)

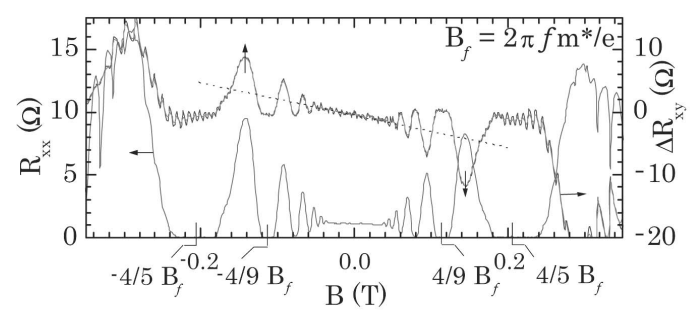

(b)

Figure 2. (a) Transport measurements with $(w /)$ and without (w/o) microwave radiation at $f=108 \mathrm{GHz}$ after Mani et al. [6]. Radiation induced vanishing $R_{x x}$ is observable around $(4 / 5) B_{f}$ and $(4 / 9) B_{f}$. A comparison of the $w /$ and $w / o$ radiation $R_{x y}$ indicates antisymmetric-in-B oscillations in $\boldsymbol{R}_{x y}$ under photoexcitation, which correlate with the $\boldsymbol{R}_{x x}$ oscillations. Here, the $w / o$ radiation Hall data have been offset for the sake of clarity; (b) The radiation induced change in the Hall resistance, $\Delta R_{x y}$, is shown along with $R_{x x}$. Note the antisymmetric-in- $B \Delta R_{x y}$ oscillations.

In the present work we shall extend our theory to Mani's phenomena. We show that the most prominent zero-resistance states observed by Mani et al. [1] and Du et al. [3] represents the integer QHE with the $v=1$ state corresponding to the superconducting state at $B=(4 / 5) B_{f}$ in Mani's case (the state $B=(2 / 3) B_{f}$ in Du's case). If we write $v=P / Q$, the Mani-series $4 /(4 j+$ 1) can be recovered by setting $j=P, Q=1$. Our model explains why the superconducting state under radiation does not accompany the Hall resistivity plateau. We predict that the fractional QHE should exist for $Q=3,5, \cdots$. The aformentioned side dip observed by Zudov et al. [5] should correspond to the state at $v=4 / 3$. This dip is missing in Mani et al.'s experiments. This is because the experimental temperature $T=0.5 \mathrm{~K}$ is so low that the dip is overshadowed by the bosonic state at $v=1$.

\section{Theory of the Quantum Hall Effect}

If the magnetic field is applied slowly, the classical electron can continuously change from the straight line motion at zero field to the curved motion at a finite $B$. 
Quantum mechanically, the change from the momentum state to the Landau state requires a perturbation. We choose for this perturebation the phonon exchange between the electron and the fluxon. Consider the cparticle with a few fluxons. If the $B$-field is applied slowly the energy of the electron does not change but the cyclotron motion always acts so as to reduce the magnetic fields. Hence the total energy of the c-particle is less than the electron energy plus the unperturbed field energy. In other words the c-particle is stable against the break-up, and it is in a bound (negative energy) state. In our theory a c-particle is simply a dressed electron carrying $Q$ fluxons. The c-particle moves as a boson (fermion) depending on the odd (even) number of fluxons in it. At the Landau level (LL) occupation number $v=1 / Q, Q$ odd, the c-bosons with $Q$ fluxons are generated, and condense below certain critical temperature $T_{c}$. The Hall resistivity plateau is due to the Meissner effect, see below.

GaAs forms a zinc blende lattice. We assume that the interface is in the plane (001). The $\mathrm{Ga}^{3+}$ ions form a square lattice with the sides directed in [110] and [1 $\overline{1} 0]$. The "electron" (wave packet) will then move isotropically with an effective mass $m_{1}$. The $\mathrm{As}^{3-}$ ions also form a square lattice at a different height in [001]. The "holes", each having a positive charge, will move similarly with an effective mass $m_{2}$. A longitudinal phonon moving in [110] or in [1 $\overline{1} 0]$ can generate a charge (current) density variations, establishing an interaction between the phonon and the electron (phonon). If one phonon exchange is considered between the electron and the fluxon, a second-order perturbation calculation establishes an effective electron-fluxon interaction [14]:

$$
V_{\text {eff }} \equiv\left|V_{q} V_{q}^{\prime}\right| \frac{\hbar \omega_{q}}{\left(\varepsilon_{|\mathbf{p}+\mathbf{q}| s}-\varepsilon_{p s}\right)^{2}-\left(\hbar \omega_{q}\right)^{2}},
$$

where $\mathbf{q}\left(\hbar \omega_{q}\right)$ is the phonon momentum (energy); $V_{q}\left(V_{q}^{\prime}\right)$ the interaction strength between the electron (fluxon) and the phonon; the Landau quantum number $N_{\mathrm{L}}$ is omitted; the bold $\mathbf{k}$ denotes the two dimensional (2D) guiding center momentum and the italic $k$ the magnitude. If the energies $\left(\varepsilon_{\mathbf{p}+\mathbf{q}}, \varepsilon_{p}\right)$ of the final and initial electron states are equal as in the degenerate LL, the effective interaction is attractive, i.e., $V_{\text {eff }}=-\left|V_{q} V_{q}^{\prime}\right| /\left(\hbar \omega_{q}\right)$.

Following Bardeen, Cooper and Schrieffer (BCS) [13], we start with a Hamiltonian $H$ with the phonon variables eliminated:

$$
\begin{aligned}
H= & \sum_{\mathbf{k}}^{\prime} \sum_{s} \varepsilon_{k}^{(1)} n_{\mathbf{k} s}^{(1)}+\sum_{\mathbf{k}}^{\prime} \sum_{s} \varepsilon_{k}^{(2)} n_{\mathbf{k s}}^{(2)}+\sum_{\mathbf{k}}^{\prime} \sum_{s} \varepsilon_{k}^{(3)} n_{\mathbf{k} s}^{(3)} \\
& -v_{0} \sum_{\mathbf{q}}^{\prime} \sum_{\mathbf{k}}^{\prime} \sum_{\mathbf{k}^{\prime}}^{\prime} \sum_{s}\left[B_{\mathbf{k}^{\prime} \mathbf{q} s}^{(1) \dagger} B_{\mathbf{k q} s}^{(1)}+B_{\mathbf{k}^{\prime} \mathbf{q} s}^{(1) \dagger} B_{\mathbf{k q} s}^{(2) \dagger}\right. \\
& \left.+B_{\mathbf{k}^{\prime} \mathbf{q} s}^{(2)} B_{\mathbf{k q} s}^{(1)}+B_{\mathbf{k}^{\prime} \mathbf{q} s}^{(2)} B_{\mathbf{k q} s}^{(2) \dagger}\right],
\end{aligned}
$$

where $n_{\mathbf{k s}}^{(j)}$ is the number operator for the "electron" (1) ["hole" (2), fluxon (3)] at momentum $\mathbf{k}$ and $\operatorname{spin} s$ with the energy $\varepsilon_{k s}^{(j)}$. We represent the "electron" ("hole") number $n_{\mathbf{k s}}^{(j)}$ by $c_{\mathbf{k s}}^{(j) \dagger} c_{\mathbf{k s}}^{(j)}$, where $c\left(c^{\dagger}\right)$ are annihilation (creation) operators satisfying the Fermi anticommutation rules:

$$
\begin{aligned}
& \left\{c_{\mathbf{k s} s}^{(i)}, c_{\mathbf{k}^{\prime} s}^{(j) \dagger}\right\} \equiv c_{\mathbf{k s} s}^{(i)} c_{\mathbf{k}^{\prime} s^{\prime}}^{(j) \dagger}+c_{\mathbf{k}^{\prime} s^{\prime}}^{(j \dagger} c_{\mathbf{k} s}^{(i)}=\delta_{\mathbf{k}, \mathbf{k}^{\prime}} \delta_{s, s^{\prime}} \delta_{i, j}, \\
& \left\{c_{\mathbf{k} s}^{(i)}, c_{\mathbf{k}^{\prime}, s^{\prime}}^{(j)}\right\}=0 .
\end{aligned}
$$

We represent the fluxon number $n_{\mathrm{k} s}^{3}$ by $a_{\mathrm{ks}}^{\dagger} a_{\mathrm{k} s}$, with $a\left(a^{\dagger}\right)$, satisfying the anticommutation rules.

$B_{\mathbf{k q} s}^{(1) \dagger} \equiv c_{\mathbf{k}+\mathbf{q} / 2 s}^{(1) \dagger} a_{-\mathbf{k}+\mathbf{q} / 2-s}^{\dagger}, \quad B_{\mathbf{k q} s}^{(2)} \equiv c_{\mathbf{k}+\mathbf{q} / 2 s}^{(2)} a_{-\mathbf{k}+\mathbf{q} / 2-s}$. The prime on the summation means the restriction: $0<\varepsilon_{k s}^{(j)}<\hbar \omega_{\mathrm{D}}$, $\omega_{\mathrm{D}}=$ Debye frequency. If the fluxons are replaced by the conduction electrons ("electrons", "holes") our Hamiltonian $H$ is reduced to the original BCS Hamiltonian, Equation (2.14) of Ref. [13]. The "electron" and "hole" are generated, depending on the energy contour curvature sign [14]. For example only "electrons" ("holes"), are generated for a circular Fermi surface with the negative (positive) curvature whose inside (outside) is filled with electrons. Since the phonon has no charge, the phonon exchange cannot change the net charge. The pairing interaction terms in Equation (2) conserve the charge. The term $-v_{0} B_{\mathbf{k}^{\prime} q}^{(1) \dagger} B_{\mathbf{k q} s}^{(1)}$, where $v_{0} \equiv\left|V_{q} V_{q}^{\prime}\right|\left(\hbar \omega_{0} A\right)^{-1}, A=$ sample area, is the pairing strength, generates the transition in the "electron" states. Similary, the exchange of a phonon generates a transition in the "hole" states, represented by $-v_{0} B_{\mathbf{k}^{\prime} \mathbf{s} s}^{(2)} B_{\mathbf{k q} s}^{(2) \dagger}$. The phonon exchange can also pair-create and pair-annihilate "electron" ("hole")fluxon composites, represented by $-v_{0} B_{\mathbf{k}^{\prime} \mathbf{s} s}^{(1) \dagger} B_{\mathbf{k q s}}^{(2) \dagger}$,

$-v_{0} B_{\mathbf{k} ' \mathbf{q} s}^{(2)} B_{\mathbf{k q s} s}^{(1)}$. At $0 \mathrm{~K}$ the system can have equal numbers of $-(+)$ c-bosons, "electrons" ("holes") composites, generated by $-v_{0} B_{\mathbf{k}^{\prime} \mathbf{q} s}^{(1 \dagger} B_{\mathbf{k q s}}^{(2) \dagger}$.

The c-bosons, each with one fluxon, will be called the fundamental (f) c-bosons. Their energies $w_{q}^{(j)}$ are obtained from [14]

$$
w_{q}^{(j)} \Psi(\mathbf{k}, \mathbf{q})=\varepsilon_{|\mathbf{k}+\mathbf{q}|}^{(j)} \Psi(\mathbf{k}, \mathbf{q})-\frac{v_{0}^{*}}{(2 \pi \hbar)^{2}} \int^{\prime} \mathrm{d}^{2} k^{\prime} \Psi\left(\mathbf{k}^{\prime}, \mathbf{q}\right),
$$

where $\Psi(\mathbf{k}, \mathbf{q})$ is the reduced wavefunction for the fcboson; we neglected the fluxon energy. The $v_{0}^{*}$ denotes the strength after the ladder diagram binding, see below. For small $q$, we obtain

$$
w_{q}^{(j)}=w_{0}+(2 / \pi) v_{\mathrm{F}}^{(j)} q, \quad w_{0}=\frac{-\hbar \omega_{\mathrm{D}}}{\exp \left(v_{0}^{*} D_{0}\right)^{-1}-1},
$$

where $v_{\mathrm{F}}^{(j)} \equiv\left(2 \varepsilon_{\mathrm{F}} / m_{j}\right)^{1 / 2}$ is the Fermi velocity and $D_{0} \equiv D\left(\varepsilon_{\mathrm{F}}\right)$ the density of states per spin. Note that the energy $w_{q}^{(j)}$ depends linearly on the momentum $q$.

The system of free fc-bosons undergoes a Bose-Eins- 
tein condensation (BEC) in 2D at the critical temperature [13]

$$
k_{\mathrm{B}} T_{\mathrm{c}}=1.24 \hbar v_{\mathrm{F}} n_{0}^{1 / 2} .
$$

The interboson distance $R_{0} \equiv n_{0}^{1 / 2}$ calculated from this expression is $1.24 \hbar v_{\mathrm{F}}\left(k_{\mathrm{B}} T_{\mathrm{c}}\right)^{-1}$. The boson size $r_{0}$ calculated from Equation (4), using the uncertainty relation $\left(q_{\max } r_{0} \sim \hbar\right)$ and $\left|w_{0}\right| \sim k_{\mathrm{B}} T_{\mathrm{c}}$, is $(2 / \pi) \hbar v_{\mathrm{F}}\left(k_{\mathrm{B}} T_{\mathrm{c}}\right)^{-1}$, which is a few times smaller than $R_{0}$. Hence, the bosons do not overlap in space, and the model of free bosons is justified. For GaAs/AlGaAs, $m=0.067 m_{\mathrm{e}}, m_{\mathrm{e}}=$ electron mass. For the 2D electron density $10^{11} \mathrm{~cm}^{-2}$, we have $v_{\mathrm{F}}=1.36 \times 10^{6} \mathrm{~cm} \cdot \mathrm{s}^{-1}$. Not all electrons are bound with fluxons since the simultaneous generations of $\pm \mathrm{fc}$-bosons is required. The minority carrier ("hole") density controls the fc-boson density. For $n_{0}=10^{10}$ $\mathrm{cm}^{-2}, T_{\mathrm{c}}=1.29 \mathrm{~K}$, which is reasonable.

In the presence of Bose condensate below $T_{\mathrm{c}}$ the unfluxed electron carries the energy $E_{k}^{(j)}=\left(\varepsilon_{k}^{(j) 2}+\Delta^{2}\right)^{1 / 2}$, where the quasi-electron energy gap $\Delta$ is the solution of

$$
\begin{aligned}
1= & v_{0} D_{0} \int_{0}^{\hbar \omega_{\mathrm{D}}} \mathrm{d} \varepsilon \frac{1}{\left(\varepsilon^{2}+\Delta^{2}\right)^{1 / 2}} \\
& \times \frac{1}{1+\exp \left[-\beta\left(\varepsilon^{2}+\Delta^{2}\right)^{1 / 2}\right]}, \quad \beta \equiv \frac{1}{k_{\mathrm{B}} T} .
\end{aligned}
$$

Note that the gap $\Delta$ depends on $T$. At $T_{\mathrm{c}}$, there is no condensate and hence $\Delta$ vanishes.

Now the moving fc-boson below $T_{\mathrm{c}}$ has the energy $\tilde{w}_{q}$ obtained from

$$
\tilde{w}_{q}^{(j)} \Psi(\mathbf{k}, \mathbf{q})=E_{|\mathbf{k}+\mathbf{q}|}^{(j)} \Psi(\mathbf{k}, \mathbf{q})-\frac{v_{0}^{*}}{(2 \pi \hbar)^{2}} \int^{\prime} \mathrm{d}^{2} k^{\prime} \Psi\left(\mathbf{k}^{\prime}, \mathbf{q}\right),
$$

where $E^{(j)}$ replaced $\varepsilon^{(j)}$ in Equation (3). We obtain

$$
\tilde{w}_{q}^{(j)}=\tilde{w}_{0}+(2 / \pi) v_{\mathrm{F}}^{(j)} q \equiv w_{0}+\varepsilon_{g}+(2 / \pi) v_{\mathrm{F}}^{(j)} q,
$$

where $\tilde{w}_{0}(T)$ is determined from

$$
1=D_{0} v_{0} \int_{0}^{\hbar \omega_{\mathrm{D}}} \mathrm{d} \varepsilon\left[\left|\tilde{w}_{0}\right|+\left(\varepsilon^{2}+\Delta^{2}\right)^{1 / 2}\right]^{-1} \text {. }
$$

The energy difference:

$$
\tilde{w}_{0}(T)-w_{0} \equiv \varepsilon_{g}(T)
$$

represents the $T$-dependent energy gap. The energy $\tilde{w}_{g}$ is negative. Otherwise, the fc-boson should break up. This limits $\varepsilon_{g}(T)$ to be $\left|w_{0}\right|$ at $0 \mathrm{~K}$. The $\varepsilon_{g}$ declines to zero as the temperature approaches $T_{\mathrm{c}}$ from below.

The fc-boson, having the linear dispersion (12), can move in all directions in the plane with the constant speed $(2 / \pi) v_{\mathrm{F}}^{(j)}$. The supercurrent is generated by the \pm fc-bosons condensed monochromatically at the momentum directed along the sample length. The supercurrent density (magnitude) $J$, calculated by the rule: $\left(\right.$ charge $\left.e^{*}\right) \times\left(\right.$ carrier density $\left.n_{0}\right) \times\left(\right.$ drift velocity $\left.v_{\mathrm{d}}\right)$, is

$$
J \equiv e^{*} n_{0} v_{\mathrm{d}}=e^{*} n_{0}(2 / \pi)\left|v_{\mathrm{F}}^{(1)}-v_{\mathrm{F}}^{(2)}\right|
$$

The induced Hall field (magnitude) $E_{\mathrm{H}}$ equals $v_{\mathrm{d}} B$. The magnetic flux is quantized $B=n_{\phi}(h / e), n_{\phi}=$ fluxon density. Hence we obtain

$$
\rho_{\mathrm{H}} \equiv \frac{E_{\mathrm{H}}}{J}=\frac{v_{\mathrm{d}} B}{e^{*} n_{0} v_{\mathrm{d}}}=\frac{1}{e^{*} n_{0}} n_{\phi}\left(\frac{h}{e}\right) .
$$

If $e^{*}=e, n_{\phi}=n_{0}$, we obtain $\rho_{\mathrm{H}}=h / e^{2}$ in agreement with the plateau value observed.

The model can be extended to the integer QHE at $v=P(Q=1)$. The field magnitude is less. The LL degeneracy $(e B A / h)$ is linear in $B$, and hence the lowest $P$ LL's must be considered. The fc-boson density $n_{0}$ per LL is the electron density $n_{\mathrm{e}}$ over $P$ and the fluxon density $n_{\phi}$ is the boson density $n_{0}$ over $P$ :

$$
n_{0}=n_{\mathrm{e}} / P, \quad n_{\phi}=n_{0} / P
$$

At $v=1 / 2$ there are c-bosons, each with two fluxons. The c-fermions have a Fermi energy. The $\pm \mathrm{c}$-fermions have effective masses. The Hall resistivity $\rho_{\mathrm{H}}$ has a $B$-linear behavior while the resistivity $\rho$ is finite.

Let us now take a general case $v=P / Q$, odd $Q$. Assume that there are $P$ sets of c-fermions with $Q-1$ fluxons, which occupy the lowest $P$ LL's. The cfermions subject to the available $B$-field form c-bosons with $Q$ fluxons. In this configuration the c-boson density $n_{0}$ and the fluxon density $n_{\phi}$ are given by Equations (18). Using Equations (17) and (18) and assuming the fractional charge $[15,16]$

$$
e^{*}=e / Q
$$

we obtain

$$
\rho_{\mathrm{H}} \equiv \frac{E_{\mathrm{H}}}{J}=\frac{v_{\mathrm{d}}}{e^{*} n_{0} v_{\mathrm{d}}} n_{\phi}\left(\frac{h}{e}\right)=\frac{Q}{P} \frac{h}{e^{2}},
$$

as observed. In our theory the integer $Q$ denotes the number of fluxons in the c-boson and the integer $P$ the number of the LL's occupied by the parental c-fermions, each with $Q-1$ fluxons.

Our Hamiltonian in Equation (6) can generate and stabilize the c-particles with an arbitrary number of fluxons. For example a c-fermion with two fluxons is generated by two sets of the ladder diagram bindings, each between the electron and the fluxon. The ladder diagram binding arises as follows. Consider a hydrogen atom. The Hamiltonian contains kinetic energies of the electron and the proton, and the attractive Coulomb 
interaction. If we regard the Coulomb interaction as a perturbation and use a perturbation theory, we can represent the interaction process by an infinite set of ladder diagrams, each ladder step connecting the electron and the proton. The energy eigenvalues of this system is not obtained by using the perturbation theory but they are obtained by solving the Schrödinger equation directly. This example indicates that a two-body bound state is represented by an infinite set of ladder diagrams and that the binding energy (the negative of the ground-state energy) is calculated by a non-perturbative method.

Jain introduced the effective magnetic field [17-19]

$$
B^{*} \equiv B-B_{v}=B-\left(\frac{1}{v}\right) n_{\mathrm{e}}\left(\frac{h}{e}\right)
$$

relative to the standard field for the composite (c-) fermion at the even-denominator fraction. We extend this to the bosonic (odd-denominator) fraction. This means that the c-particle moves field-free at the exact fraction. The c-particle is viewd as the quasiparticle containing an electron circulating around $Q$ fluxons. The jumping of the guiding centers (the $\mathrm{CM}$ of the c-particle) can occur as if they are subject to no $B$-field at the exact fraction. The excess (or deficit) of the magnetic field is simply the effective magnetic field $B$. The plateau in $\rho_{\mathrm{H}}$ is formed due to the Meissner effect. Consider the case of zero temperature near $v=1$. Only the energy $E$ matters. The fc-bosons are condensed with the ground-state energy $w_{0}$, and hence the system energy $E$ at $v=1$ is $2 N_{0} w_{0}$, where $N_{0}$ is the number of -fc-bosons (or + fc-bosons). The factor 2 arises since there are $\pm f c-$ bosons. Away from $v=1$, we must add the magnetic field energy $\left(2 \mu_{0}\right)^{-1} A\left(B^{*}\right)^{2}$, so that

$$
E=2 N_{0} w_{0}+\left(2 \mu_{0}\right)^{-1} A\left(B^{*}\right)^{2} .
$$

When the field is reduced, the system tries to keep the same number $N_{0}$ by sucking in the flux lines. Thus the magnetic field becomes inhomogeneous outside the sample, generating the magnetic field energy

$\left(2 \mu_{0}\right)^{-1} A\left(B^{*}\right)^{2}$. If the field is raised, the system tries to keep the same number $N_{0}$ by expeling out the flux lines. The inhomogeneous fields outside raise the field energy as well. There is a critical field $B_{c}^{*}=\left(4 \mu_{0}\left|w_{0}\right|\right)^{1 / 2}$. Beyond this value, the superconducting state is destroyed, generating a symmetric exponential rise in $R$. In our discussion of the Hall resistivity plateau we used the fact that the ground-state energy $w_{0}$ of the fc-boson is negative, that is, the c-boson is bound. Only then the critical field $B_{c}^{*}=\left(4 \mu_{0}\left|w_{0}\right|\right)^{1 / 2}$ can be defined. Here the phonon exchange attraction played an important role. The repulsive Coulomb interaction, which is the departure point of the prevalent theories [12], cannot generate a bound state.
In the presence of the supercondensate the noncondensed c-boson has an energy gap $\varepsilon_{g}$. Hence the noncondensed c-boson density has the activation energy type exponential temperature-dependence:

$$
\exp \left[-\varepsilon_{g} /\left(k_{\mathrm{B}} T\right)\right] \text {. }
$$

In the prevalent theories the energy gap for the fractional QHE is identified as the sum of the creation energies of a quasi-electron and a quasi-hole [20-23]. With this view it is difficult to explain why the activationenergy type temperature dependence shows up in the steady-state quantum transport. Some authors argue that the energy gap $\varepsilon_{g}$ for the integer QHE is due to the LL separation $=\hbar \omega_{0}$. But the separation $\hbar \omega_{0}$ is much greater than the observed $\varepsilon_{g}$. Besides from this view one cannot obtain the activation-type energy dependence.

The BEC occurs at each LL, and therefore the c-boson density $n_{0}$ is less for high $P$, see Equation (18), and the strengths become weaker as $P$ increases.

\section{Quantum Hall Effect under Radiation}

The experiments by Mani et al. [6] indicate that the applied radiation excites a large number of "holes" in the system. Using these "holes" and the preexisting "electrons" the phonon exchange can pair-create $\pm c$-bosons, which condense below $T_{\mathrm{c}}$ in the excited channel. The c-bosons condensed with the momentum along the sample length are responsible for the supercurrent. In the presence of the condensed c-bosons, the non-condensed c-bosons have an energy gap $\varepsilon_{g}$, and therefore they are absent at $0 \mathrm{~K}$. The c-fermions in the base channel have the energies $E_{p}=\left(\varepsilon_{p}^{2}+\Delta^{2}\right)^{2}$ but their energy spectra have no gap. Hence they are not completely suppressed at the lowest temperatures. They contribute a small normal current. They are subject to the Lorentz force: $\mathbf{F}=q(\mathbf{E}+\mathbf{v} \times \mathbf{B})$, and they generate a Hall field $E_{\mathrm{H}}$ proportional to the field $B$. Figure 2 show that the deviation in the Hall resistance, $\Delta R_{x y} \equiv R_{x y}-R_{x y, \text { dark }}$, is closely correlated to the resistance $R_{x x}$. We shall demonstrate this behavior based on the two channel (carriers) model.

In the neighbarhood of the principal QHE at $v=j=1$ the carriers in the base and excited channels are respectively c-fermions and c-bosons condensed. The currents are additive. We write down the total current density $j$ as the sum of the fermionic current density $j_{\mathrm{f}}$ and the bosonic $j_{\mathrm{b}}$ :

$$
j=j_{\mathrm{f}}+f_{\mathrm{b}}=e n_{\mathrm{f}} v_{\mathrm{f}}+e n_{\mathrm{b}} v_{\mathrm{b}},
$$

where $v_{\mathrm{f}}$ and $v_{\mathrm{b}}$ are the drift velocities of the fermions and bosons. The Hall fields $E_{\mathrm{H}}$ are additive, too. Hence we have

$$
E_{\mathrm{H}}=E_{\mathrm{H}, \mathrm{f}}+E_{\mathrm{H}, \mathrm{b}}=v_{\mathrm{f}} B+v_{\mathrm{b}} B .
$$


We note that the Hall effect condition $\left(E_{\mathrm{H}}=v_{\mathrm{d}} B\right)$ applies for the fermions and bosons. We therefore obtain

$$
R_{\mathrm{H}}=\frac{E_{\mathrm{H}}}{j}=\frac{v_{\mathrm{f}} B+v_{\mathrm{b}} B}{n_{\mathrm{f}} v_{\mathrm{f}}+n_{\mathrm{b}} v_{\mathrm{b}}} \cdot \frac{1}{e} .
$$

Far away from the midpoint of the zero-resistance stretch the c-bosons are absent and hence the Hall resistivity $R_{\mathrm{H}}$ becomes $B /\left(e n_{\mathrm{f}}\right)$ after the cancellation of $v_{\mathrm{f}}$. At the midpoint the c-bosons are dominant. Then, the Hall resistivity $R_{\mathrm{H}}$ is approximately $h / e^{2}$ since

$$
\frac{E_{\mathrm{H}}}{j} \cong \frac{v_{\mathrm{b}} B}{e n_{\mathrm{b}} v_{\mathrm{b}}} \cong \frac{h}{e^{2}} \frac{n_{\phi}}{n_{\mathrm{b}}}=\frac{h}{e^{2}},
$$

where we used the flux quantization $\left[B=(h / e) n_{\phi}\right]$, and the fact that the flux density $n_{\phi}$ equals the c-boson density $n_{\mathrm{b}}$. The Hall resistivity $R_{\mathrm{H}}=E_{\mathrm{H}} / j$ is not exactly equal to $h / e^{2}$ since the c-fermion current density $e n_{\mathrm{f}} v_{\mathrm{f}}$ is much smaller than the supercurrent density $e n_{\mathrm{b}} v_{\mathrm{b}}$, but it does not vanish. In the horizontal stretch the system is superconducting, and hence the supercurrent dominates the normal current: $e n_{\mathrm{b}} v_{\mathrm{b}} \gg e n_{\mathrm{f}} v_{\mathrm{f}}$. The deviation $\Delta R_{\mathrm{H}}$ is, using Equation (26),

$$
\Delta R_{\mathrm{H}}=\frac{v_{\mathrm{f}} B+v_{\mathrm{b}} B}{e\left(n_{\mathrm{f}} v_{\mathrm{f}}+n_{\mathrm{b}} v_{\mathrm{b}}\right)}-\frac{v_{\mathrm{f}} B}{e n_{\mathrm{f}} v_{\mathrm{f}}} \simeq \frac{v_{\mathrm{b}} B}{e n_{\mathrm{b}} v_{\mathrm{b}}} \simeq \frac{h}{e^{2}} .
$$

If the field $B$ is raised (or lowered) a little from the midpoint, $\Delta R_{\mathrm{H}}$ is a constant $\left(h / e^{2}\right)$ due to the Meissner effect. If the field is raised high enough, the superconducting state is destroyed and the normal current sets in, generating a finite resistance and a vanishing $\Delta R_{\mathrm{H}}$. Hence the deviation $\Delta R_{\mathrm{H}}$ and the diagonal resistance $R_{x x}$ are closely correlated.

In Figure 2 we observe that in the range where the Shubnikov-de Haas (ShdH) oscilations are observed for the resistance without radiation, the signature of oscillations also appear for the resistance $R_{x x}$ with radiation. The ShdH oscillation arise only for the fermion carriers. The fermionic currents cannot be suppressed by the supercurrents. This ShdH signature in $R_{x x}$ should remain. Hence our two-channel model is supported.

Mani et al.'s experiments, Figure 2 of Ref. [1], show that the strength of the superconducting state does not change much for the radiation frequency $\omega$ in the range $(47,110) \mathrm{GHz}$. This feature may come as follows. The 2D density of states for the conduction electrons associated with the circular Fermi surface is independent of the electron energy, and hence the number of the excited electrons is roughly independent of the radiation energy (frequency). The "hole"-like excitations are absent with no radiation. We suspect that the "hole"-band edge is a distance $\varepsilon_{0}$ away from the system's Fermi level. This means that if the radiation energy $\hbar \omega$ is less than $\varepsilon_{0}$, the radiation can generate no superconducting state. This feature can be checked by applying radiation of frequencies lower than $47 \mathrm{GHz}$. Mani's experiments on the simultaneous radiation excitations suggest that the critical frequency is between 15 and $47 \mathrm{GHz}$.

In summary the QHE under radiation is the QHE at the upper channel. The condensed c-bosons generate a superconducting state with a gap $\varepsilon_{g}$ in the c-boson energy spectrum. The supercondensate changes the c-fermion energy from $\varepsilon_{k}$ to $\left(\varepsilon_{k}^{2}+\Delta^{2}\right)^{1 / 2}$ in the base channel. This energy spectrum has no gap, and hence the cfermions cannot be suppressed completely at the lowest temperatures, and generate a finite resistive current accompanied by the Hall field. This explains the $B$-linear Hall resistivity. Our microscopic theory can be tested experimentally by examining 1) the "hole"-like excitations by a circularly polarized laser; 2 ) the bosonic state at $v=4 / 3$ and $1 / 3 ; 3)$ the "hole" band edge.

\section{REFERENCES}

[1] R. G. Mani, J. H. Smet, K. von Klitzing, V. Narayanamurti, W. B. Johnson and V. Umansky, "Zero-Resistance States Induced by Electromagnetic-Wave Excitation in GaAs/AlGaAs Heterostructures," Nature, Vol. 420, 2004, pp. 646-650. doi:10.1038/nature01277

[2] R. G. Mani, "Zero-Resistance States Induced by Electromagnetic-Wave Excitation in GaAs/AlGaAs Heterostructures," Physica E, Vol. 22, 2004, pp. 1-6. doi:10.1016/j.physe.2003.11.204

[3] R. R. Du, M. A. Zudov, C. L. Yang, Z. Q. Yuan, L. N. Pfeiffer and K. W. West, "Oscillatory and Vanishing Resistance States in Microwave Irradiated 2D Electron Systems," In: Y. Wang, L. Engel and N. Bonesteel, Eds., High Magnetic Fields in Semiconductor Physics, World Scientific, Singapore, 2005, pp. 11-18. doi:10.1142/9789812701923_0001

[4] D. C. Tsui, H. L. Störmer and A. C. Gossard, "Two- Dimensional Magnetotransport in the Extreme Quantum Limit," Physical Review Letters, Vol. 48, 1982, pp. 15591562. doi:10.1103/PhysRevLett.48.1559

[5] M. A. Zudov, R. R. Du, L. N. Pfeiffer and K. W. West, "Evidence for a New Dissipationless Effect in 2D Electronic Transport," Physical Review Letters, Vol. 90, 2003, Article ID: 046807. doi:10.1103/PhysRevLett.90.046807

[6] R. G. Mani, V. Narayanamurti, K. von Klitzing, J. H. Smet, W. B. Johnson and V. Umansky, "Radiation-Induced Oscillatory Hall Effect in Highmobility GaAs/ AlxGa1 xAs Devices," Physical Review B, Vol. 69, 2004, Article ID: 161306. doi:10.1103/PhysRevB.69.161306

[7] J. C. Phillips, "Microscopic Origin of Collective Exponentially Small Resistance States," Solid State Communications, Vol. 127, No. 3, 2003, pp. 233-236. doi:10.1016/S0038-1098(03)00350-8

[8] A. V. Andreev, I. L. Aleiner and A. J. Millis, "Dynamical Symmetry Breaking as the Origin of the Zero-dc-Resistance State in an ac-Driven System," Physical Review Letters, Vol. 91, No. 5, 2003, Article ID: 056803. 
doi:10.1103/PhysRevLett.91.056803

[9] A. C. Durst, S. Sachdev, N. Read and S. M. Girvin, "Radiation-Induced Magnetoresistance Oscillations in a 2D Electron Gas," Physical Review Letters, Vol. 91, No. 8, 2003, Article ID: 086803. doi:10.1103/PhysRevLett.91.086803

[10] J. Shi and X. C. Xie, "Radiation-Induced Zero-Resistance State and the Photon-Assisted Transport," Physical Review Letters, Vol. 91, No. 8, 2003, Article ID: 086801. doi:10.1103/PhysRevLett.91.086801

[11] F. S. Bergeret, B. Huckestein and A. F. Volkov, "CurrentVoltage Characteristics and the Zero-Resistance State in a Two-Dimensional Electron Gas," Physical Review B, Vol. 67, 2003, Article ID: 241303. doi:10.1103/PhysRevB.67.241303

[12] S. Fujita, S. Godoy and D. Nguyen, "Bloch Electron Dynamics," Foundation of Physics, Vol. 25, No. 8, 1995, pp. 1209-1220. doi:10.1007/BF02055258

[13] J. Bardeen, L. N. Cooper and J. R. Schrieffer, "Theory of Superconductivity," Physical Review, Vol. 108, No. 5, 1957, pp. 1175-1204. doi:10.1103/PhysRev.108.1175

[14] S. Fujita, Y. Tamura and A. Suzuki, "Microscopic Theory of the Quantum Hall Effect," Modern Physics Letters B, Vol. 15, No. 20, 2001, pp. 817-825. doi:10.1142/S0217984901002610

[15] R. B. Laughlin, “Anomalous Quantum Hall Effect: An
Incompressible Quantum Fluid with Fractionally Charged Excitations," Physical Review Letters, Vol. 50, No. 18, 1983, pp. 1395-1398. doi:10.1103/PhysRevLett.50.1395

[16] F. D. M. Haldane, "Fractional Quantization of the Hall Effect: A Hierarchy of Incompressible Quantum Fluid States," Physical Review Letters, Vol. 51, No. 7, 1983, pp. 605-608. doi:10.1103/PhysRevLett.51.605

[17] J. K. Jain, "Composite-Fermion Approach for the Fractional Quantum Hall Effect," Physical Review Letters, Vol. 63, No. 2, 1989, pp. 199-202. doi:10.1103/PhysRevLett.63.199

[18] J. K. Jain, "Incompressible Quantum Hall States," Physical Review B, Vol. 40, No. 11, 1989, pp. 8079-8082. doi:10.1103/PhysRevB.40.8079

[19] J. K. Jain, “Theory of the Fractional Quantum Hall effect," Physical Review B, Vol. 41, No. 11, 1990, pp. 76537665. doi:10.1103/PhysRevB.41.7653

[20] R. E. Prange and S. M. Girvin, "The Quantum Hall Effect," 2nd Edition, Springer-Verlag, New York, 1990.

[21] Z. F. Ezawa, "Quantum Hall Effects," World Scientific, Singapore, 2000.

[22] M. Stone, "Quantum Hall Effect," World Scientific, Singapore, 1992.

[23] T. Chakraborty and P. Pietilainen, "Quantum Hall Effects," 2nd Edition, Springer-Verlag, Berlin, 1995. doi:10.1007/978-3-642-79319-6 